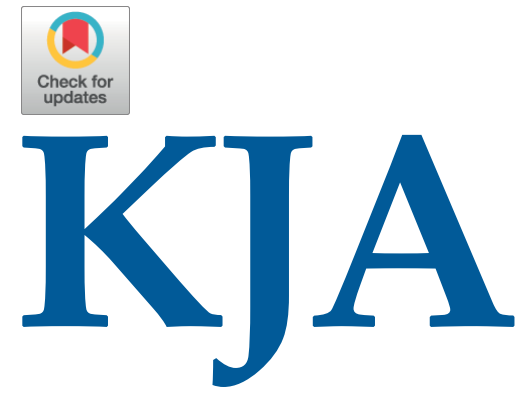

Korean Journal of Anesthesiology

\section{Clinical Research Article}

Korean J Anesthesiol 2021;74(5):449-458

https://doi.org/10.4097/kja.21133

pISSN 2005-6419• eISSN 2005-7563

Received: March 26, 2021

Revised: May 31, 2021 (1st); July 9, 2021

(2nd)

Accepted: August 4, 2021

Corresponding author:

Jun Hyun Kim, M.D., Ph.D.

Department of Anesthesiology and Pain

Medicine, Inje University Ilsan Paik Hospital,

170 Juhwa-ro, Ilsanseo-gu, Goyang 10380,

Korea

Tel: +82-31-910-7067

Fax: +82-31-910-7184

Email: ishmael@paik.ac.kr

ORCID: https://orcid.org/0000-0002-9466-8399

*Min Hee Heo and Ji Yeon Kim contributed equally and shared the first authorship to this study.

\title{
Epidural analgesia versus intravenous analgesia after minimally invasive repair of pectus excavatum in pediatric patients: a systematic review and meta- analysis
}

\author{
Min Hee Heo*, Ji Yeon Kim*, Jung Hyeon Kim, Kyung Woo Kim, \\ Sang Il Lee, Kyung-Tae Kim, Jang Su Park, Won Joo Choe, \\ Jun Hyun Kim
}

Department of Anesthesiology and Pain Medicine, Inje University Ilsan Paik Hospital, Goyang, Korea

Background: Postoperative pain control after the minimally invasive repair of pectus excavatum (MIRPE) is essential, but there is a controversy about a better analgesic method between epidural and intravenous (IV) analgesia. This systematic review and meta-analysis aimed to compare the effect of epidural versus IV analgesia following MIRPE.

Methods: We searched PubMed, MEDLINE, Embase, Cochrane Central Register, and ClinicalTrials.gov for randomized controlled trials (RCTs) dated up to 31st May 2021. The primary outcome was the area under the curve (AUC) of the weighted mean visual analog scale (VAS) after MIRPE. The secondary outcomes were postoperative nausea, operation time, total operating room time, and postoperative length of hospital stay.

Results: Four RCTs involving 243 patients were finally included in this meta-analysis. The AUC of the weighted mean VAS was 343.62 in the epidural group and 375.24 in the IV group. The epidural group showed lower VAS than the IV group at 12 to $48 \mathrm{~h}$ after the surgery. Postoperative nausea, operation time and length of hospital stay was not different between two groups. The epidural group had a significantly longer total operating room time due to epidural catheter insertion time.

Conclusions: Epidural analgesia after the MIRPE had a better analgesic effect than IV analgesia. However, IV analgesia may also be a viable option, and physicians should wisely choose analgesic modalities after MIRPE.

Keywords: Epidural analgesia; Funnel chest; Intravenous administration; Minimally invasive surgical procedures; Postoperative pain; Statistics; Systematic review; Thoracic surgery.

\section{Introduction}

Pectus excavatum is a relatively common deformity that occurs in nearly 1 in 1,000 children [1]. In 1998, Donald Nuss introduced a minimally invasive repair of pectus excavatum (MIRPE) called the Nuss procedure or pectus bar procedure [2]. In this procedure, a substernal bar is positioned in the chest through small axillary incisions. It is a standard treatment and is less invasive than the open Ravitch procedure that it replaced. Operation time is short and blood loss is minor, but postoperative pain is not minimal $[3,4]$. Postoperative pain control after MIRPE has a significant effect on the length of hospital stay [5]. 
The pathophysiology of pain after the procedure has not been fully evaluated, but the primary source of pain is the chest wall's stretching caused by the substernal bar, not the incisions [6]. Previous studies have suggested that thoracic epidural analgesia is the most effective method for controlling pain during the early postoperative period [6-8]. However, thoracic epidural analgesia requires an experienced anesthesiologist. Complications, such as infection, nerve damage, and epidural hematoma, may occur; and the procedure can prolong the operation time [9]. According to the results of a recent randomized controlled trial (RCT), intravenous (IV) patient-controlled analgesia (PCA) had the same effect as epidural analgesia up to postoperative day 3 [10]. Also, Gasior et al. [11] compared the effect of epidural analgesia and IV analgesia on MIRPE patients' long-term perceptions of their pain control experiences after an average of 3.2 years. The results did not show any significant difference in perceptions between the two methods.

The aim of our systematic review and meta-analysis was to compare the effect of epidural analgesia and IV analgesia in MIRPE patients.

\section{Materials and Methods}

\section{Study design}

This systematic review and meta-analysis were performed according to the recommendations of the PRISMA (Preferred Reporting Items for Systematic Reviews and Meta-Analyses) and Cochrane Collaboration [12,13]. The protocol was preregistered in PROSPERO (https://www.crd.york.ac.uk/PROSPERO, no. CRD42020169362).

\section{Search strategy}

Two trained reviewers, K.J.H. and L.S.I., independently searched PubMed, MEDLINE, Embase, the Cochrane Central Register of Controlled Trials, and ClinicalTrials.gov for studies that had been completed but not published before May 31, 2021 without language restriction. The following keywords were searched: 'Nuss', 'Pectus excavatum,' 'Epidural,' and 'Patient-controlled analgesia.' The search strategy is presented in the Supplementary Materials.

\section{Study selection and eligibility criteria}

The reviewers independently screened studies first by title and abstract and then by the full text. They assessed full-text articles separately, and any disagreements were resolved through discus- sion with the third author (K.J.Y.).

Studies to be included in the analysis had to have met the following Population, Intervention, Comparison, Outcomes and Study (PICOS) criteria. Population: patients who received MIRPE; Interventions: Epidural PCA or block or infusion (epidural group); Comparison intervention: IV analgesics including PCA (IV group); Outcome: Primary outcome could be measured using a visual analog scale (VAS) or numeric rating scale (NRS), and the secondary outcomes could be postoperative nausea, operation time, total operating room time, postoperative length of stay in the hospital, number of calls made to anesthesiologists, and number of hours until the patient could consume a regular diet; Study design: RCTs. Observational studies, non-randomized studies, and quasi-randomized studies were excluded.

\section{Risk of bias in individual studies}

The methodological quality of the studies was assessed using the Revised Cochrane Risk-of-bias tool [12]. Two reviewers (K.J.H., L.S.I.) evaluated the methodological quality of the included studies. For the evaluation of the risk of bias, we used the Cochrane methodology. According to Cochrane's five items, we assessed each study separately as low, high, or some concerns risk of bias [12].

\section{Data extraction}

The two trained authors (K.J.H., L.S.I.) independently extracted data from the articles and cross-checked them to avoid extracting incorrect information. The extracted information included the authors' names, publication year, patients' ages, patient gender ratio, number of patients in the studies, treatments they received, clinical setting information, follow-up duration, and outcome data. We contacted the corresponding authors of the studies via e-mail for which NRS or VAS data was missing or more information was needed. When pain scores were not presented in numbers, we extracted pain scores using graphs or figures.

\section{Statistical analysis}

The studies' pain score results were measured and compared using the area under the curve (AUC) of the VAS from postoperative hour 0 to hour 108. The AUC of the mean VAS was calculated for included studies that had individual participants' pain scores and the AUC of the weighted mean VAS was calculated otherwise [14]. These calculations were performed using MedCalc v.19.6.1 (MedCalc Software, Belgium) for Windows. 
Forest plots of summarized pain scores at postoperative hours 0, 12, 24, 48, 72, and 96 are presented. The Hartung, Knapp, Sidik, and Jonkman (HKSJ) method was used to reduce type I errors because the number of included studies was small [15].

If standard deviations were not reported, we used an average of the standard deviations from the other studies that reported those [16]. For articles that only reported the median and interquartile range, the Median-IQR method was used to impute the mean as the median and the SD as the third quartile to the first quartile [17]. Publication bias was checked by examining funnel plots, but Egger's test could not be used because fewer than five studies were included. Analysis was performed with RevMan v.5.3 (Review Manager, The Nordic Cochrane Centre, The Cochrane Collaboration, Denmark) and the metafor package v.2.4.0 for R (R Foundation for Statistical Computing, Austria). This analysis was performed using a fixed-effects model to conduct a meta-analysis in the absence of significant heterogeneity (defined as $\mathrm{P}>0.10$ and $\left.\mathrm{I}^{2}<40 \%\right)$ and a random-effects model otherwise.

\section{Results}

Our search strategy identified 893 records (Fig. 1). The list of the excluded articles and the reasons for their exclusion are presented in Supplementary Table 1. Four studies were eligible for the final analyses. The characteristics of the included studies are presented in Table 1 and Supplementary Table $2[7,10,18$, 19]. Standard deviations were not reported in two articles, so the average of the standard deviations from the other articles that did was used instead [18,19]. Visual inspection of the funnel plots did not reveal significant publication bias (Supplementary Fig. 1). The overall risk of bias was some concerns for three articles $[7,18,19]$, low for one article [10] (Fig. 2).

The AUC of the weighted mean VAS was 343.62 in the epidural group and 375.24 in the IV group, indicating that the epidural group had a lower cumulative pain score than the IV group (Fig. 3) $[7,10,18,19]$.

There was no difference in VAS between the epidural and IV groups at postoperative hour 0 (4 RCTs, epidural group $\mathrm{n}=121$, IV group $\mathrm{n}=122$, mean difference $[\mathrm{MD}]:-0.91,95 \%$ CI $[-2.11$, 0.29 ], $\mathrm{P}=0.138, \mathrm{I} 2=51 \%$ ) (Fig. $4 \mathrm{~A}$ ). The epidural analgesia group had a lower VAS than the IV analgesia group at postoperative hours 12 (4 RCTs, epidural group $\mathrm{n}=121$, IV group $\mathrm{n}=122$, MD: $-0.99,95 \%$ CI: [-1.52, -0.47], P = 0.001, $\mathrm{I}^{2}=0 \%$ ) (Fig. 4B), 24 (4 RCTs, epidural group $\mathrm{n}=121$, IV group $\mathrm{n}=122, \mathrm{MD}$ : $-0.65,95 \%$ CI $[-1.15,-0.16], \mathrm{P}=0.009, \mathrm{I}^{2}=0 \%$ ) (Fig. 4C), and 48 (4 RCTs, epidural group $\mathrm{n}=121$, IV group $\mathrm{n}=122, \mathrm{MD}$ : $-0.81,95 \%$ CI $[-1.61,-0.01], \mathrm{P}=0.046, \mathrm{I}^{2}=46 \%$ ) (Fig. 4C), and
48 (4 RCTs, epidural group $\mathrm{n}=121$, IV group $\mathrm{n}=122$, MD: -0.81 [95\% CI: $-1.61,-0.01$ ] $, \mathrm{P}=0.046, \mathrm{I}^{2}=46 \%$ ) (Fig. 4D) [7,10,18,19].

At postoperative hours 72 and 96, there were no differences in the VAS in the epidural and IV groups (Figs. 4E and 4F) $[7,10,18]$.

Three articles reported on the secondary outcome, postoperative nausea, and there was no difference between them (Fig. 5) $[7,18,19]$. Operation time did not differ between the epidural and IV groups (Fig. 6) [7,10,18,19]. The epidural group had a significantly longer total operating room time than the IV group, including epidural catheter insertion time (Fig. 7) [10,18]. The groups did not have different postoperative hospital stay lengths (Fig. 8) $[7,10,18]$. Significantly fewer calls to anesthesiologists were made for the IV group than the epidural group (Supplementary Fig. 2) [10,18]. There was no difference between the groups in the number of hours until patients could eat a regular diet (Supplementary Fig. 3) [10,18].

About the epidural catheter insertion failure, Weber's study reported that there was no technical difficulty [7]. Six patients (11\%)

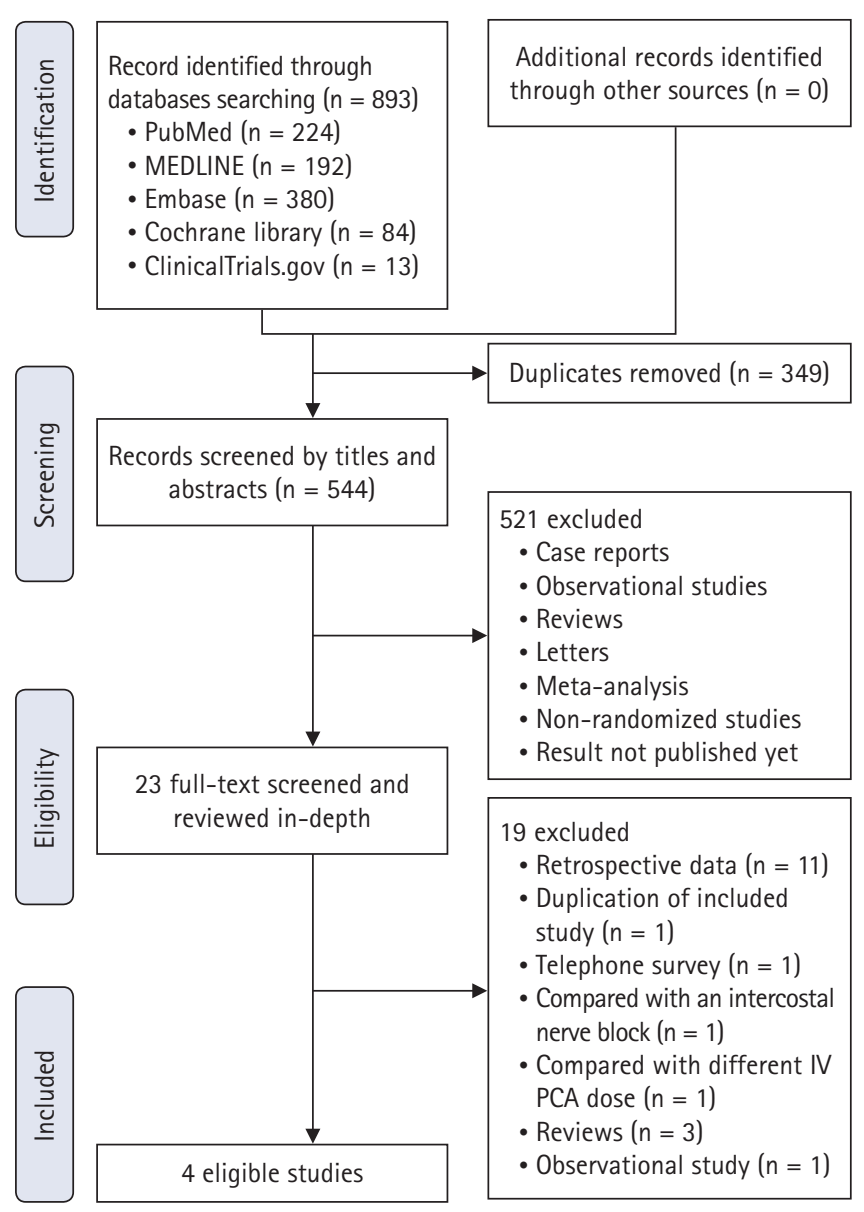

Fig. 1. Flow chart of study selection. 
Table 1. The Characteristics of Included Studies [7,10,18,19].

\begin{tabular}{|c|c|c|c|c|c|c|}
\hline & $\begin{array}{l}\text { Total patients } \\
\text { (n) (Epi/IV) }\end{array}$ & Mean age \pm SD & $\begin{array}{l}\text { Gender, } \\
\text { male (\%) }\end{array}$ & Reported outcomes & Follow-up period & $\begin{array}{l}\text { Epidural catheter } \\
\text { removal }\end{array}$ \\
\hline Butkovic 2007 [19] & $28(14 / 14)$ & 14.5 & 75 & $\begin{array}{l}\text { Pain score (VAS)*, operation time*, } \\
\text { the level of sedation, heart rate, } \\
\text { systolic/diastolic arterial blood } \\
\text { pressure, ventilator frequency, } \\
\mathrm{PaO}_{2}, \mathrm{PaCO}_{2} \text {, oxygen saturation, } \\
\text { side effects (nausea* pruritus) }\end{array}$ & $\begin{array}{l}\text { During the first } 48 \\
\text { h after surgery }\end{array}$ & N/A \\
\hline Weber 2007 [7] & $40(20 / 20)$ & $15.9 \pm 4.7$ & 80 & $\begin{array}{l}\text { Pain score (VAS)*, operation time*, } \\
\text { postoperative length of stay*, sub- } \\
\text { jective well-being, intraoperative } \\
\text { fentanyl, side effects (sedation, } \\
\text { nausea*, pruritus) }\end{array}$ & $\begin{array}{l}\text { At the end of anes- } \\
\text { thesia }(0 \mathrm{~h}) \text {, every } \\
12 \mathrm{~h} \text { until } 96 \mathrm{~h} \\
\text { postoperative time }\end{array}$ & POD 4 (96h) \\
\hline St Peter 2012 [18] & $110(55 / 55)$ & $15.5 \pm 2.9$ & N/A & $\begin{array}{l}\text { Pain score* (VAS, the average of } \\
\text { sitting and supine pain scores), } \\
\text { operation time*, total operating } \\
\text { room time*, postoperative length } \\
\text { of stay*, hospital course (calls to } \\
\text { anesthesia*, hours to regular diet*, } \\
\text { hours to foley catheter removal, } \\
\text { hours to oral medications, proce- } \\
\text { dure charges, anesthesia charges, } \\
\text { nausea*, total hospital charges) }\end{array}$ & $\begin{array}{l}\text { POD 0-5, twice } \\
\text { daily (AM, PM) }\end{array}$ & POD $2.8 \pm 0.5$ \\
\hline Sujka 2020 [10] & $65(32 / 33)$ & $14.75 \pm 1.35$ & 92 & $\begin{array}{l}\text { Pain score* (NRS, rest, and dynamic } \\
\text { pain), operation time*, total oper- } \\
\text { ating room time*, calls to anesthe- } \\
\text { sia*, hours to regular diet*, postop- }^{*} \text { - } \\
\text { erative length of stay* }\end{array}$ & $\begin{array}{l}\text { POD 0-4, twice } \\
\text { daily (AM, PM) }\end{array}$ & POD 3 \\
\hline
\end{tabular}

Epi: epidural group, IV: intravenous group, SD: standard deviation, VAS: visual analogue scale, POD: postoperative day, NRS: numeric rating scale, $\mathrm{PaO}_{2}$ : partial pressure of oxygen, $\mathrm{PaCO}_{2}$ : partial pressure of carbon dioxide, N/A: not available. ${ }^{*}$ The outcomes were analyzed in this study.

$\begin{array}{lcccccc} & \frac{D 1}{!} & \frac{\text { D1 }}{!} & \frac{\text { D1 }}{+} & \underline{\text { D1 }} & \underline{\text { D1 }} & \underline{\text { Overall }} \\ \text { Butkovic 2007 } & ! & ! & + & + & + & ! \\ \text { Weber 2007 } & ! & + & + & + & + & ! \\ \text { St Peter 2012 } & ! & + & + & + & + & ! \\ \text { Sujka 2020 } & + & + & + & + & + & +\end{array}$

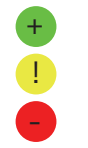

Low risk

Some concerns

High risk

D1

D2

D3

D4

D5

Randomisation process

Deviations from the intended interventions

Missing outcome data

Measurement of the outcome

Selection of the reported result

Fig. 2. Assessment of methodological quality of included studies based on the revised Cochrane risk-of-bias tool (RoB 2) for RCTs [7,10,18,19]. RCT: randomized controlled trials.

in St Peter's study and three patients (9\%) in Sujka's study suffered epidural catheter insertion failure $[10,18]$. About the inadequate epidural analgesia, six patients (11\%) in St Peter's study had their epidural catheters removed within $24 \mathrm{~h}$ after surgery and nine patients (28\%) in Sujka's study required IV PCA due to inadequate analgesia $[10,18]$. Two studies reported the incidence of unintended sedation, and there were no significant differences between the groups [7,19]. Pruritis was only reported in Butkovic's study. Three patients $(21.4 \%)$ in the epidural group experienced it while none in the IV group did [19].

\section{Discussion}

This study compared epidural analgesia and IV analgesia at managing pain after MIRPE in terms of the AUC of weighted mean VAS. The epidural group's VAS AUC was 343.62, which was lower than the IV group's 375.24. The epidural group's VAS was lower than that of the IV group from postoperative hour 12 to 48, but there was no difference in analgesic effect between the groups from postoperative hour 0 to 12 or after 72 .

Thoracic epidural analgesia has been recommended as a gold standard for postoperative analgesia after MIRPE [20,21]. Muhly et al. [6] reported that $91 \%$ of surveyed institutions reported that they used epidural analgesia to manage pain after MIRPE and 


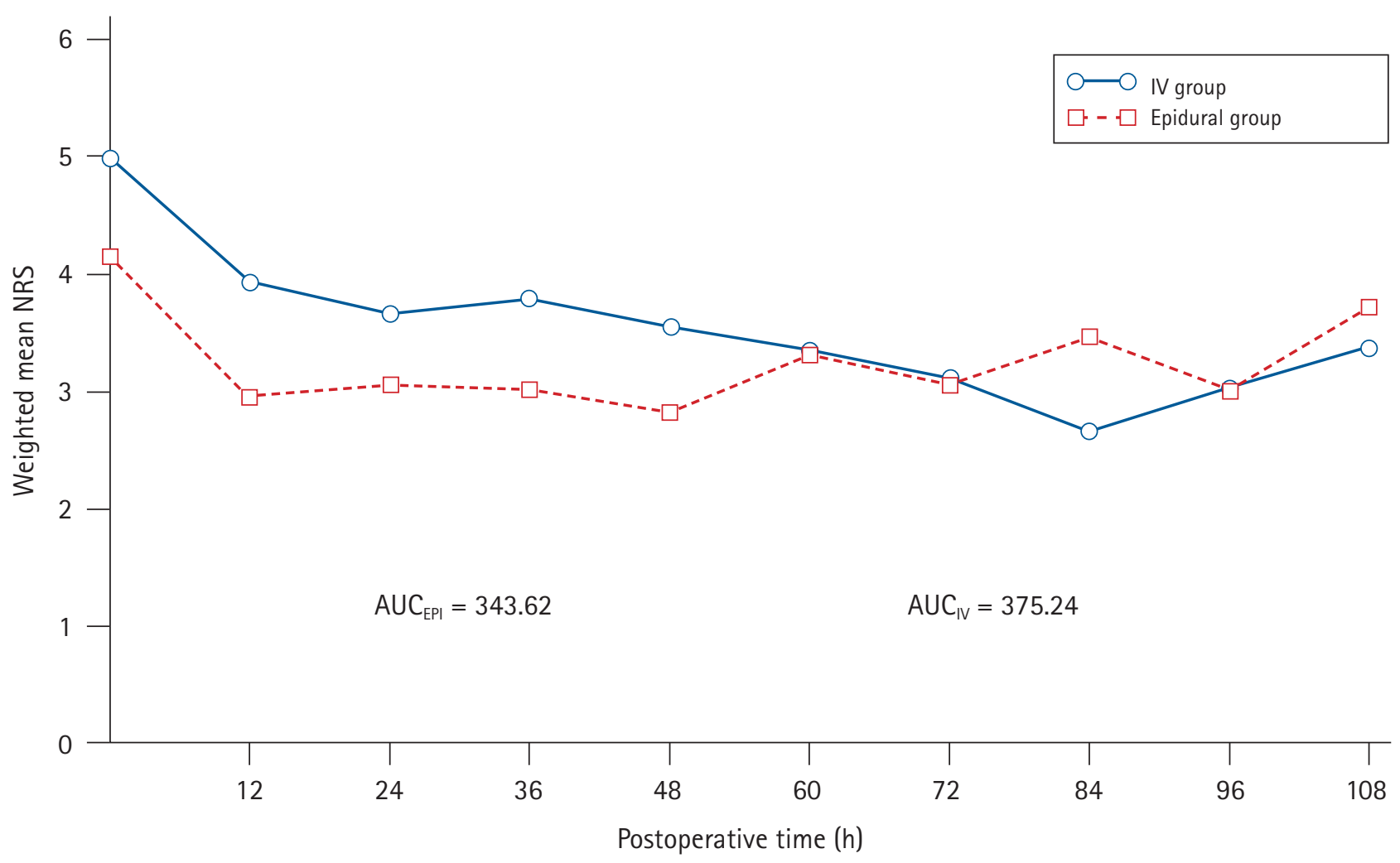

Fig. 3. Weighted mean VAS of epidural group or IV group [7,10,18,19]. VAS: visual analog scale, NRS: numeric rating scale, AUC: area under the curve, IV: intravenous, Epi: epidural.

$27 \%$ use epidural and IV analgesia in combination. However, recently, a study found that $16 \%$ of the institutions do not use epidural analgesia at surgeons' request due to concerns about neurological complications [6,9]. Thoracic epidural catheter insertion fails in $2 \%$ of pediatric patients and catheter-related complications, such as dislodgement and kinking, occur in $8 \%$ [22]. Of the studies included this article, epidural catheter insertion failure rates were $0 \%, 9 \%$, and $11 \%$, respectively $[7,10,18]$. When including patients who required IV analgesia because of inadequate epidural analgesia, the failure rate increased to 35\% [18]. Even in adult patients, epidurals fail for various reasons, such as insufficient analgesia or catheter dislodgement, in 32\% of patients [23].

There are concerns about epidural-related complications, as well as concerns about failure. Even when a well-trained anesthesiologist performs thoracic epidural catheter placement, unexpected complications can occur. In children, epidural-related complications, most of which are due to infections and medication errors, occur in 6 out of every 1,000 cases. Permanent neurological damage had also been reported to occur in 1 in every 10,000 cases $[24,25]$. Neurological complications included hematoma, trauma to the spinal cord or dura, and prolonged motor blockages $[4,9,25]$. These complications occur at a higher rate among pediatric patients than adult patients because pediatric patients are less willing to cooperate during epidural catheter insertion without sedation, so detection of neurological complications after the procedure may be delayed because the patients have to be sedated [26].

In addition to these concerns, there are several other limitations to using epidural analgesia. Epidural catheter insertion takes time, as shown in this meta-analysis. Despite the time required for and difficulties of inserting epidural catheters, they are usually removed within three days due to concerns about infection. This meta-analysis showed that epidural catheters were removed at the latest by postoperative days $3-4[7,10,14]$. There are concerns about postoperative nausea when using IV analgesia, but in this study, its incidence was similar in both groups. Also, in the retrospective review of St Peter et al. [5], the epidural group had a longer postoperative hospital stay than the IV group by $15 \mathrm{~h}(\mathrm{P}=$ 0.037), but in this study, both groups had similar stays. The difference found in their study might have been caused by the need to administer oral analgesic medication to the epidural group after removing their catheters [5].

Despite the limitations of epidural analgesia, the epidural group in this study had a low weighted mean VAS AUC, suggesting that 


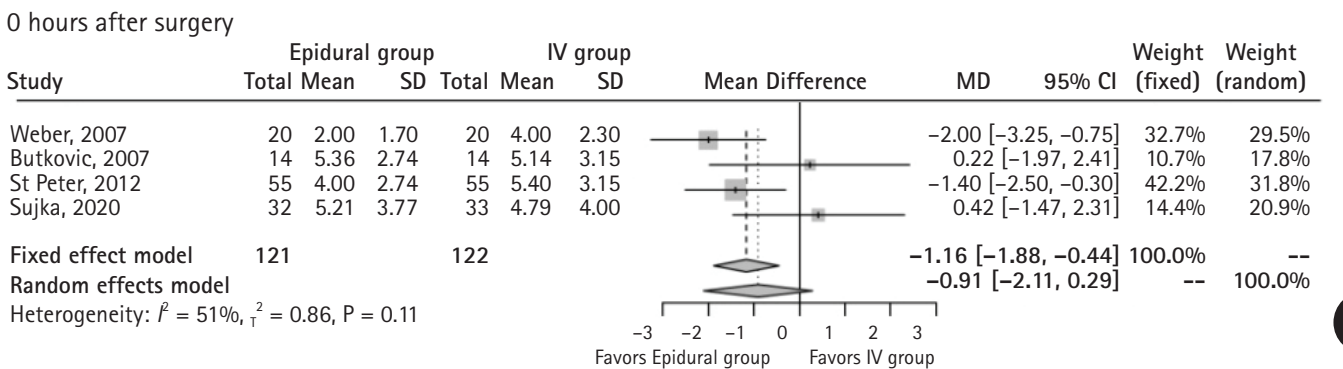

12 hours after surgery

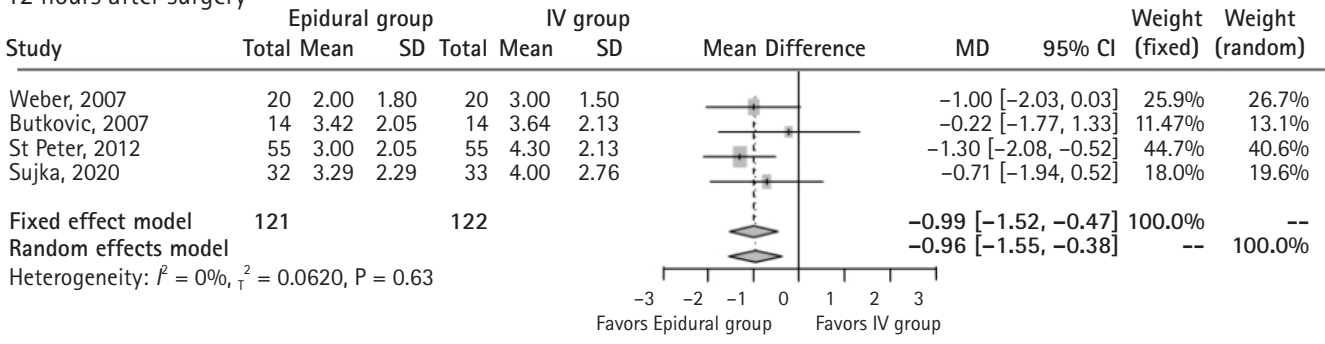

24 hours after surgery

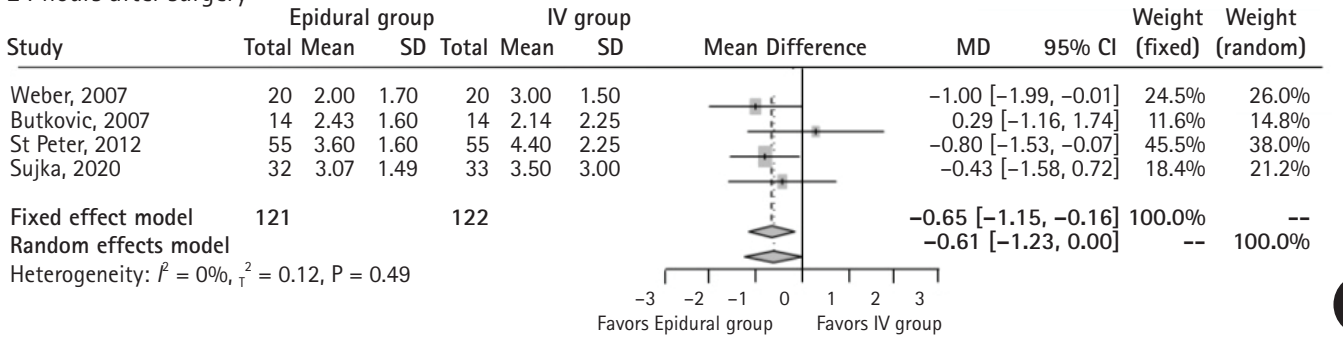

48 hours after surgery

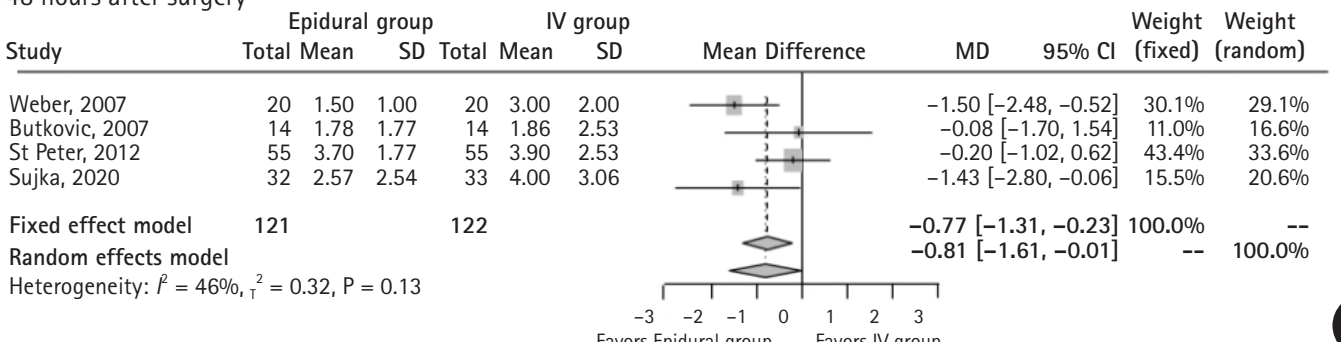

72 hours after surgery

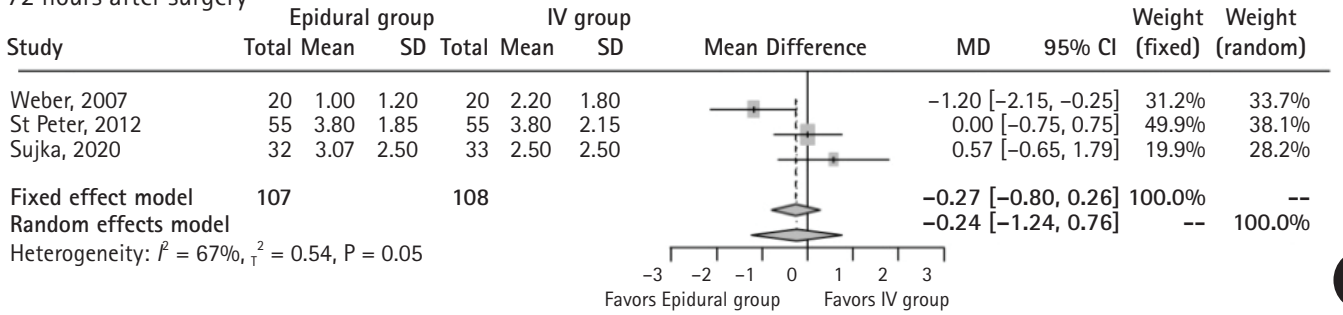

96 hours after surgery

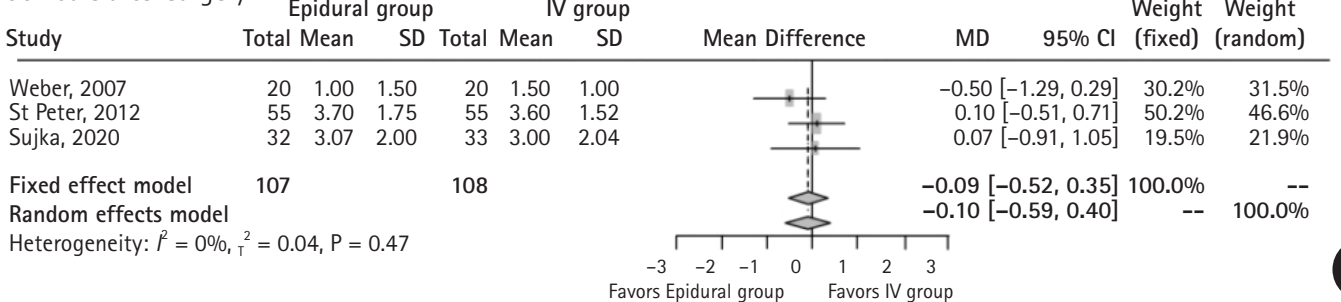

Fig. 4. Forest plot of primary outcome data (VAS) for (A) 0 h, (B) 12 h, (C) 24 h, (D) 48 h, (E) 72 h, and (F) $96 \mathrm{~h}$ after surgery [7,10,18,19]. VASSD: standard deviation, VAS: visual analog scale. 


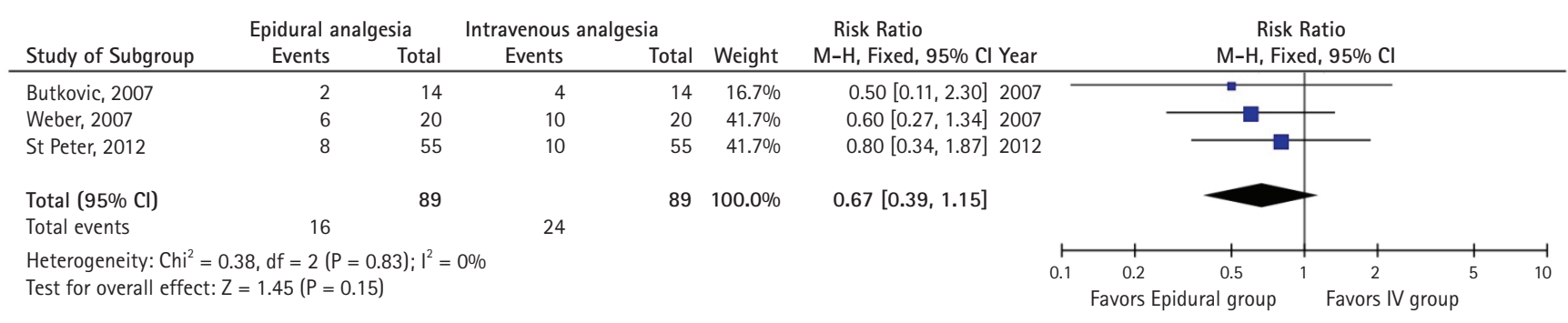

Fig. 5. Forest plot of postoperative nausea [7,18,19]. The event numbers in the Weber 2007 study [7] was calculated from the graph presented in Fig. 3.D of the article.

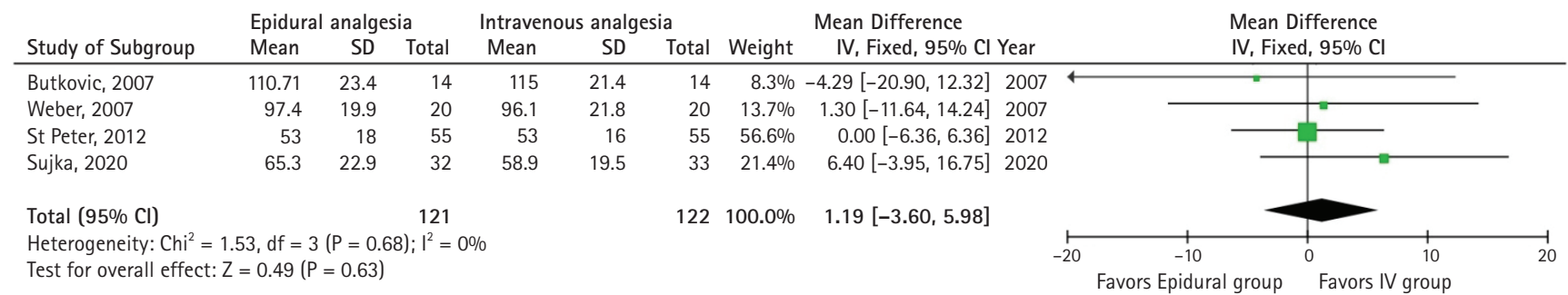

Fig. 6. Forest plot of operation time (min) $[7,10,18,19]$.

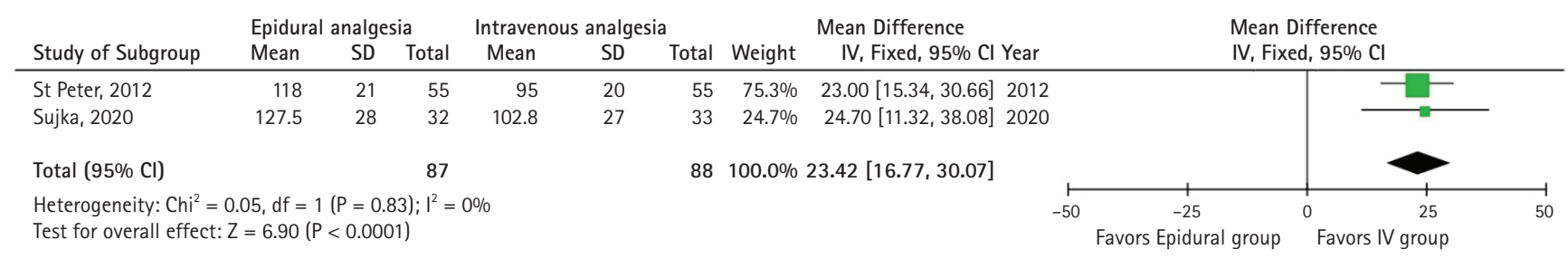

Fig. 7. Forest plot of total operating room time (min) [10,18].

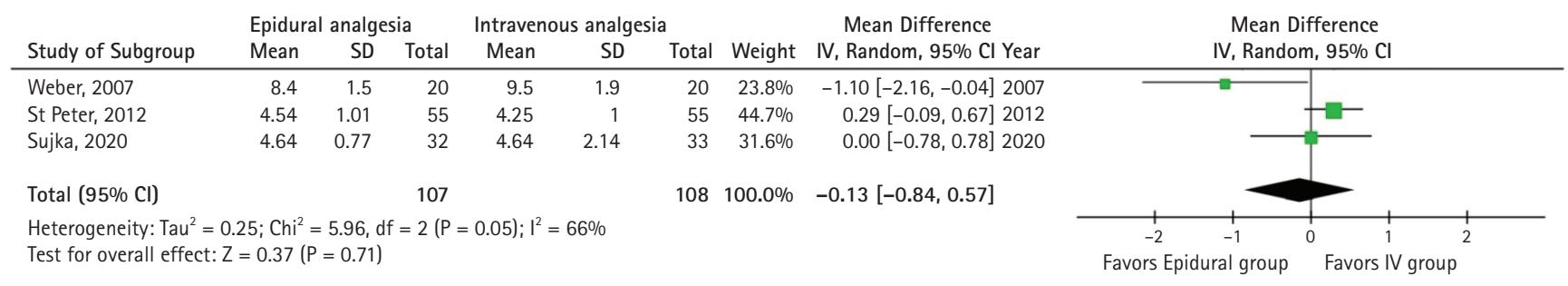

Fig. 8. Forest plot of postoperative length of stay (day) $[7,10,18]$.

epidural analgesia has a strong analgesic effect after MIRPE. Even when the weighted mean VAS AUC was analyzed without restricting the types of studies, it was shown to be also lower in the epidural group than the IV group (Supplementary Fig. 4) $[7,8,10,18,19,27]$. The epidural group had a lower pain score than the IV group from postoperative hours 12 to 48 (Fig. 4). This result may have been a product of the fact that epidural catheters were removed on postoperative days 3-4. Before removal, the postoperative analgesic effect was higher in the epidural group than the IV group. Interestingly, the pain score did not signifi- cantly differ immediately after surgery (Fig. 4A). This result was similar to that of the previous meta-analysis [28], and our analysis which includes RCTs and retrospective studies altogether (Supplementary Fig. 4). This result may have been a product of the fact that the patient population was children in our study and previous meta-analysis who might not accurately express their pain levels. The remaining analgesic effect of the anesthetics might have affected the VAS.

Uncontrolled postoperative pain causes several problems in children and adolescents who account for most MIRPE patients. 
Pain can cause adverse physiological and psychological outcomes, such as decreased immune response, disturbed sleep, decreased physical functioning, anxiety, chronic and persistent post-surgical pain, and psychiatric problems [29]. Epidural analgesia after MIRPE can prevent respiratory depression sparing systemic narcotics and can last for the duration of three days that the epidural catheter is in place. Adequate postoperative analgesia enables early ambulation, coughing, and deep breathing, thus preventing atelectasis [5]. Therefore, physicians should be familiar with the characteristics of epidural and IV analgesia and choose between them appropriately.

The limitations of this study were as follows. First, only four RCTs met the inclusion criteria. Second, this meta-analysis can be seen as an update of a prior meta-analysis because it added Sujka et al's study [10,28]. Third, although the AUC of the weighted mean VAS was calculated, its statistical significance could not be calculated because the relevant raw data could not be retrieved.

Notwithstanding these limitations, this study had several significant strengths and unique qualities. First, the AUC of weighted mean VAS was calculated to analyze pain relief over time instead of just using the VAS for a specific time. Unfortunately, individual patient data meta-analysis could not be performed as was originally intended because the articles identified in this study did not include raw data. However, by comparing the groups' weighted mean VAS's AUCs, it was possible to compare pain management during the postoperative period. Second, the previous meta-analysis showed that the epidural had a lower pain score than the IV group at postoperative hour 0 , but this study showed that there was no significant difference between the groups. In this study, the Hartung, Knapp, Sidik, and Jonkman method was used instead of the DerSimonian and Laird method that was used in the previous meta-analysis because it produces fewer type I errors than the DerSimonian and Laird method for small numbers of studies [15]. Third, only RCTs were analyzed to increase the results' reliability. Even though only four articles were included, Cochrane does not recommend that a mix of observational studies and RCTs be used for meta-analyses [12]. Fourth, the search for articles in this study was conducted without language limitations to maximize search results. Fifth, forest plots for secondary outcomes, such as postoperative nausea, operation time, total operating room time, postoperative length of hospital stay, the number of calls to anesthesiologists, and the number of hours until patients could eat a regular diet, were analyzed. Thus, this study produced a more comprehensive understanding of how to choose between epidural and IV analgesia after MIRPE.

In conclusion, the epidural group had better pain control as reflected by a lower cumulative AUC value of the weighted mean
VAS than the IV group until postoperative hour 108 and a lower VAS for postoperative hours 12 to 48 . However, IV analgesia may still be a viable option because epidural analgesia has higher rates of failure and complications and a high conversion rate to IV analgesia. Therefore, physicians should carefully choose between these pain control methods after MIRPE by considering their patients' conditions, their hospital's usual practice, and their skill.

\section{Conflicts of Interest}

No potential conflict of interest relevant to this article was reported.

\section{Author Contributions}

Min Hee Heo (Formal analysis; Writing - original draft; Writing - review \& editing)

Ji Yeon Kim (Conceptualization; Methodology; Supervision; Writing - original draft; Writing - review \& editing)

Jung Hyeon Kim (Data curation; Validation)

Kyung Woo Kim (Data curation; Investigation; Resources)

Sang Il Lee (Conceptualization; Methodology; Resources)

Kyung-Tae Kim (Resources)

Jang Su Park (Methodology)

Won Joo Choe (Supervision)

Jun Hyun Kim (Conceptualization; Formal analysis; Methodology; Supervision; Writing - review \& editing)

\section{Supplementary Materials}

Search strategy

Supplementary Table 1. Table of the excluded studies.

Supplementary Table 2 . The characteristics of included studies in detail.

Supplementary Fig. 1. Funnel plots of the primary outcome of pain scores.

Supplementary Fig. 2. Forest plot of calls to anesthesiologists.

Supplementary Fig. 3. Forest plots of hour to a regular diet.

Supplementary Fig. 4. AUC graph of weighted mean VAS of epidural group and IV group including two retrospective studies which met our inclusion criteria except study type, and data could be retrieved $[1,2]$.

\section{ORCID}

Min Hee Heo, https://orcid.org/0000-0002-2469-8445

Ji Yeon Kim, https://orcid.org/0000-0002-4686-5638 
Jung Hyeon Kim, https://orcid.org/0000-0002-9488-4606

Kyung Woo Kim, https://orcid.org/0000-0003-1447-9385

Sang Il Lee, https://orcid.org/0000-0003-0315-1659

Kyung-Tae Kim, https://orcid.org/0000-0002-3032-8109

Jang Su Park, https://orcid.org/0000-0002-6125-1079

Won Joo Choe, https://orcid.org/0000-0001-7564-9451

Jun Hyun Kim, https://orcid.org/0000-0002-9466-8399

\section{References}

1. Goretsky MJ, Kelly RE Jr, Croitoru D, Nuss D. Chest wall anomalies: pectus excavatum and pectus carinatum. Adolesc Med Clin 2004; 15: 455-71.

2. Nuss D, Kelly RE Jr, Croitoru DP, Katz ME. A 10-year review of a minimally invasive technique for the correction of pectus excavatum. J Pediatr Surg 1998; 33: 545-52.

3. Densmore JC, Peterson DB, Stahovic LL, Czarnecki ML, Hainsworth KR, Davies HW, et al. Initial surgical and pain management outcomes after Nuss procedure. J Pediatr Surg 2010; 45 : 1767-71.

4. Mavi J, Moore DL. Anesthesia and analgesia for pectus excavatum surgery. Anesthesiol Clin 2014; 32: 175-84.

5. St Peter SD, Weesner KA, Sharp RJ, Sharp SW, Ostlie DJ, Holcomb GW 3rd. Is epidural anesthesia truly the best pain management strategy after minimally invasive pectus excavatum repair? J Pediatr Surg 2008; 43: 79-82.

6. Muhly WT, Maxwell LG, Cravero JP. Pain management following the Nuss procedure: a survey of practice and review. Acta Anaesthesiol Scand 2014; 58: 1134-9.

7. Weber T, Mätzl J, Rokitansky A, Klimscha W, Neumann K, Deusch E; Medical Research Society. Superior postoperative pain relief with thoracic epidural analgesia versus intravenous patient-controlled analgesia after minimally invasive pectus excavatum repair. J Thorac Cardiovasc Surg 2007; 134: 865-70.

8. Soliman IE, Apuya JS, Fertal KM, Simpson PM, Tobias JD. Intravenous versus epidural analgesia after surgical repair of pectus excavatum. Am J Ther 2009; 16: 398-403.

9. Meyer MJ, Krane EJ, Goldschneider KR, Klein NJ. Case report: neurological complications associated with epidural analgesia in children: a report of 4 cases of ambiguous etiologies. Anesth Analg 2012; 115: 1365-70.

10. Sujka JA, Dekonenko C, Millspaugh DL, Doyle NM, Walker BJ, Leys CM, et al. Epidural versus PCA pain management after pectus excavatum repair: a multi-institutional prospective randomized trial. Eur J Pediatr Surg 2020; 30: 465-71.

11. Gasior AC, Weesner KA, Knott EM, Poola A, St Peter SD. Longterm patient perception of pain control experience after partici- pating in a trial between patient-controlled analgesia and epidural after pectus excavatum repair with bar placement. J Surg Res 2013; 185: 12-4.

12. Higgins JP, Thomas J, Chandler J, Cumpston M, Li T, Page MJ, et al. Cochrane Handbook for Systematic Reviews of Interventions version 6.2 [Internet]. London: Cochrane [updated $2021 \mathrm{Feb}$; cited 2021 Mar 26]. Available from www.training.cochrane.org/ handbook.

13. Moher D, Liberati A, Tetzlaff J, Altman DG; PRISMA Group. Preferred reporting items for systematic reviews and meta-analyses: the PRISMA statement. PLoS Med 2009; 6: e1000097.

14. Cappelleri JC, Bushmakin AG, Zlateva G, Sadosky A. Pain responder analysis: use of area under the curve to enhance interpretation of clinical trial results. Pain Pract 2009; 9: 348-53.

15. IntHout J, Ioannidis JP, Borm GF. The Hartung-Knapp-Sidik-Jonkman method for random effects meta-analysis is straightforward and considerably outperforms the standard DerSimonian-Laird method. BMC Med Res Methodol 2014; 14: 25.

16. Furukawa TA, Barbui C, Cipriani A, Brambilla P, Watanabe N. Imputing missing standard deviations in meta-analyses can provide accurate results. J Clin Epidemiol 2006; 59: 7-10.

17. Greco T, Biondi-Zoccai G, Gemma M, Guerin C, Zngrillo A, Landoni G. How to impute study-specific standard deviations in meta-analyses of skewed continuous endpoints? World J Metaanal 2015; 3: 215-24.

18. St Peter SD, Weesner KA, Weissend EE, Sharp SW, Valusek PA, Sharp RJ, et al. Epidural vs patient-controlled analgesia for postoperative pain after pectus excavatum repair: a prospective, randomized trial. J Pediatr Surg 2012; 47: 148-53.

19. Butkovic D, Kralik S, Matolic M, Kralik M, Toljan S, Radesic L. Postoperative analgesia with intravenous fentanyl PCA vs epidural block after thoracoscopic pectus excavatum repair in children. Br J Anaesth 2007; 98: 677-81.

20. Semmelmann A, Kaltofen H, Loop T. Anesthesia of thoracic surgery in children. Paediatr Anaesth 2018; 28: 326-31.

21. McBride WJ, Dicker R, Abajian JC, Vane DW. Continuous thoracic epidural infusions for postoperative analgesia after pectus deformity repair. J Pediatr Surg 1996; 31: 105-7.

22. Polaner DM, Taenzer AH, Walker BJ, Bosenberg A, Krane EJ, Suresh S, et al. Pediatric Regional Anesthesia Network (PRAN): a multi-institutional study of the use and incidence of complications of pediatric regional anesthesia. Anesth Analg 2012; 115: 1353-64.

23. Hermanides J, Hollmann MW, Stevens MF, Lirk P. Failed epidural: causes and management. Br J Anaesth 2012; 109: 144-54.

24. Jöhr M. Regional anaesthesia in neonates, infants and children: 
an educational review. Eur J Anaesthesiol 2015; 32: 289-97.

25. Walker BJ, Long JB, Sathyamoorthy M, Birstler J, Wolf C, Bosenberg AT, et al. Complications in pediatric regional anesthesia: an analysis of more than 100,000 blocks from the pediatric regional anesthesia network. Anesthesiology 2018; 129: 721-32.

26. Llewellyn N, Moriarty A. The national pediatric epidural audit. Paediatr Anaesth 2007; 17: 520-33.

27. Reinoso-Barbero F, Fernández A, Durán P, Castro LE, Campo G, Melo MM. Thoracic epidural analgesia vs patient-controlled analgesia with intravenous fentanyl in children treated for pectus excavatum with the Nuss procedure. Rev Esp Anestesiol Reanim 2010; 57: 214-9.

28. Stroud AM, Tulanont DD, Coates TE, Goodney PP, Croitoru DP. Epidural analgesia versus intravenous patient-controlled analgesia following minimally invasive pectus excavatum repair: a systematic review and meta-analysis. J Pediatr Surg 2014; 49: 798806.

29. Cunningham ME, Vogel AM. Analgesia, sedation, and delirium in pediatric surgical critical care. Semin Pediatr Surg 2019; 28: 33-42. 